\title{
Full-Size ABC Transporters from the ABCG Subfamily in Medicago truncatula
}

\author{
Michal Jasinski, ${ }^{1}$ Joanna Banasiak, ${ }^{1}$ Marcin Radom, ${ }^{2}$ Anna Kalitkiewicz, ${ }^{3}$ and Marek Figlerowicz ${ }^{1}$ \\ ${ }^{1}$ Institute of Bioorganic Chemistry PAS, Noskowskiego 12/14, 61-704 Poznań, Poland; ${ }^{2}$ Institute of Computing Science, \\ Poznań Institute of Technology, Piotrowo 2, 60-965 Poznań, Poland; ${ }^{3}$ Department of Plant Biotechnology, Faculty of Natural \\ Science, University of Szczecin, Waska 13, 71-415 Szczecin, Poland
}

Submitted 9 December 2008. Accepted 15 April 2009.

Full-size ATP-binding cassette (ABC) transporters belonging to the ABCG subfamily are unique for plants and fungi. There is growing evidence that certain of these proteins play a role in plant defense or signaling systems. As yet, a complete set of full-size ABCG protein genes has been inventoried and classified in only two plants: Arabidopsis thaliana and Oryza sativa. Recently, a domain-based clustering analysis has predicted the presence of at least 12 genes encoding such proteins in the Lotus japonicus genome. Here, we identify and classify 19 genes coding fullsize ABCG proteins in Medicago truncatula, a model legume plant. We have found that the majority of these genes are expressed in roots and flowers whereas only a few are expressed in leaves. Expression of several has been induced upon pathogenic infection in both roots and leaves. ABCG messenger RNAs have been detected in root nodules forming during symbiosis of legume plants and nitrogen-fixing bacteria. The data presented provide a scaffold for further studies of the physiological function of Medicago ABCG transporters and their possible role in modulating plantmicroorganism interactions.

ATP-binding cassette (ABC) proteins occur in all living species. Most of them are involved in transmembrane transport of a great variety of molecules. All members of this protein family have a characteristic modular organization. They are composed of one or two copies of two basic structural elements: a hydrophobic transmembrane domain (TMD) and a cytosolic part containing an $\mathrm{ABC}$ transporter-specific nucleotide-binding domain (NBD) (Crouzet et al. 2006; Verrier et al. 2008). Based on two characteristic features, the number and the spatial distribution of structural domains, $\mathrm{ABC}$ transporters were divided into several subfamilies. The ABCG subfamily is composed of two major types of proteins: i) half-size transporters, formerly named white-brown complex homologs, comprising only one NBD and TMD in so-called reverse orientation with the NBD domain approaching the amino terminal end of the protein (NBDTMD); and ii) full-size ABCG transporters, earlier known as plant pleitropic drug-resistant (PDR)-like subfamily, containing two NBD and two TMD (NBD-TMD $)_{2}$. The latter probably arose by a single duplication of the ancestral half-size ABCG genes (Cruzet et al. 2006).

Corresponding author: Marek Figlerowicz; Telephone: +48-61-852-85-03; Fax. +48-61-852-05-32; E-mail: marekf@ibch.poznan.pl

* The $\boldsymbol{e}$-Xtra logo stands for "electronic extra" and indicates that four supplementary tables and two supplementary figures are published online.
A detailed phylogenetic analysis of $\mathrm{ABC}$ transporters shows that plant full-size ABCG proteins form clearly separated clusters. Parallel genomic studies demonstrate that full-size ABCG proteins are widespread in plants; 15 members have been identified in Arabidopsis and more than 20 in rice (Verrier et al. 2008). Recently collected data revealed the great functional diversity of plant full-size ABCG proteins. In addition, they provide new evidence that members of the $A B C G$ family are involved in plant response to biotic and abiotic stresses. For instance, RNAi-mediated NpPDR1 silencing caused Nicotiana plumbaginifolia to be more sensitive to infection with pathogenic fungus Botrytis cinerea (Stukkens et al. 2005). Moreover, it has been shown that the expression of this gene is upregulated by sclareol (a secondary metabolite with antimicrobial properties), a potential NpPDR1 substrate (Jasinski et al. 2001). Similarly, knockdown of the PDR8/PEN3 transporter in Arabidopsis increased plant susceptibility to bacterial and oomycete pathogens (Kobae et al. 2006; Stein et al. 2006). Other genes belonging to the same subfamily are induced either by pathogens and elicitors derived from them or by plant hormones (e.g. salicylic acid, methyl jasmonate, or ethylene). Abiotic stress such as high salt concentration, cold, presence of lead, or iron deficiency also induces the expression of fullsize ABCG proteins (Crouzet et al. 2006). The number of ABC proteins and, in particular, full-size transporters in plants exceeds that reported in other multicellular organisms. This reflects different adaptive strategies of plants and, possibly, a higher number of potential substrates.

Although legumes are the third largest plant family in the world and the second most important crop family; our knowledge concerning legume ABC transporters is limited. One of them, GmPDR12, was identified in soybean (Glycine max) suspension cell cultures. The expression of this gene has been rapidly induced by salicylic acid but, to date, little is known about GmPDR12 expression and function in planta (Eichhorn et al. 2006). Recently, several partial sequences of full-size ABCG transporters have been found in Lotus japonicus. Based on the conserved domain assembly, the authors identified 12 putative full-size ABCG genes. A few of those are induced in roots upon symbiotic infection (Sugiyama et al. 2006).

Here, we identify and classify 19 full-size ABCG transporters (MtABCG) from barrel medic (Medicago truncatula Gaertn.). All of them contain distinctive PDR motives and have been classified together with other known full-size ABCG proteins. The profiles of $M t A B C G$ expression have been determined. Most of them are transcribed in roots $(n=14)$ and flowers $(n=11)$. Few transcripts have been found in leaves. Moreover, we identified MtABCG genes being upregulated upon pathogenic fungal infection in roots and leaves. 


\section{RESULTS}

\section{Identification of the full-size ABCG transporter genes} in M. truncatula.

Although the analysis of the expressed sequence tag (EST) indicated the expression of several different members of the ABCG subfamily in $M$. truncatula, to date, those transporters were not systematically classified. To solve this problem, we made a search for the MtABCG genes in all currently available genomic databases. The size of the $M$. truncatula genome was estimated to be 450 to $560 \mathrm{Mbp}$ (Medicago Genome Sequencing Consortium 2006). The first release of the genomic sequence (186.2 Mbp; Mt 1.0 database) contained between 55 and 58\% of all genes captured by sequence assembly ( $M$. truncatula release 1.0; Medicago Genome Sequencing Consortium 2006). A current release (214.7 Mbp; Mt 2.0 database), raises the percentage of coverage of the protein coding sequences to more than $60 \%$.

In order to identify genes encoding transporters of interest, we performed a BlastP analysis on the translated products. As a query, we used the most conserved fragments of the PDR motifs: i) GLDARAA and TIHQPS, placed in carboxyl terminal part of ABCG proteins; and ii) LLLGPP and GLDSST, located in the amino terminal part (Ducos et al. 2005; Rea 2007; van den Brûle and Smart 2002). As a result, we have identified 19 full-size ABCG transporters with at least three PDR motifs in the predicted sequence (Table 1). Because the average full-size ABCG protein is composed of 1,400 amino acids (Rea 2007), we took into account only sequences longer than 700 amino acids. Within shorter fragments, we found a few members of WBC subfamily of halfsize ABCG transporters (e.g., IMGA|CT573078_29.5. and IMGA|AC146751_46.4) but these were not classified here. All 19 identified $\bar{M}$. truncatula ABCG transporters were used for the construction of the phylogenetic tree. The latter also included the previously described complete sequences of full-size ABCG proteins from Arabidopsis thaliana, rice (Oryza sativa), soybean (G. max), spirodela (Spirodela polyrrhiza), and Nicotiana spp. (Fig. 1). The proteins were named according to the recently proposed unified nomenclature (Supplementary Table S1) (Verrier et al. 2008).
At the current status of genome sequencing, $M$. truncatula displays a high number of ABCG proteins, comparable with that of rice and higher than Arabidopsis. Overall sequence identity between $M$. truncatula ABCG proteins is 50 to $70 \%$; however, several of them (e.g., MtABCG1 to MtABCG4 and MtABCG9 to MtABCG11) form tight clades with sequence identity up to 91 and $98 \%$, respectively (Supplementary Table S2). High similarity between these proteins is also reflected in the conserved organization of their gene structures (Supplementary Figure S1). Moreover, we noticed that one group of close homologs, MtABCG9 to MtABCG11, displays 70\% amino acid identity to AtABCG40 from Arabidopsis. Such a high gene redundancy may indicate that duplication of some full-size MtABCG has occurred in M. truncatula as a result of an adaptation to a new function. Interestingly, a close homolog of MtABCG1 to MtABCG4 proteins is the GmPDR12 (GmABCG1) transporter from soybean, sharing $82 \%$ amino acid identity with MtABCG1. This identity is higher than any other observed between, for instance, M. truncatula and Arabidopsis ABCG proteins (Supplementary Table S3), suggesting a "legume" nature of this clade and possible divergence that occurred after Fabaceae appearance. Similar species-specific clustering of certain ABCG genes is observed for Arabidopsis (e.g. AtABCG30 and 41 to AtABCG43) or rice (OsABCG32 and OsABCG34 to OsABCG37) and has been proposed to be related to acquisition of new functions or substrates (Crouzet et al. 2006).

\section{Full-size ABCG genes expression in M. truncatula.}

Initially, the accumulation of MtABCG messenger (m)RNAs was analyzed by the reverse-transcriptase polymerase chain reaction (RT-PCR) method. The specific primers were designed and preliminary tests involving genomic DNA allowed us to set an optimal PCR condition (Supplementary Table S4). Obtained products were sequenced, confirming primer specificity (not shown). Only two genes, MtABCG9 and MtABCG11, sharing $95 \%$ nucleotide sequence identity (including the $3^{\prime}$ untranslated region), were not distinguishable by the PCR method and, therefore, the same pair of primers was applied for their identification. The obtained set of primers was used for the RT-PCR analysis of MtABCG mRNA accumulation in differ-

Table 1. Details of the Medicago truncatula ABCG genes

\begin{tabular}{|c|c|c|c|c|c|c|c|c|}
\hline Name & $\begin{array}{c}\text { TC } \\
\text { number, } \\
\text { TIGR }\end{array}$ & $\begin{array}{c}\text { Name, } \\
\text { IMGAG }\end{array}$ & $\begin{array}{l}\text { Name, } \\
\text { MIPS }\end{array}$ & $\begin{array}{l}\text { Size } \\
\text { (bp) }\end{array}$ & $\begin{array}{l}\text { Spliced } \\
\text { size } \\
\text { (bp) }\end{array}$ & $\begin{array}{c}\text { Size } \\
\text { (amino } \\
\text { acids) }\end{array}$ & $\mathbf{P D R}^{\mathbf{a}}$ & Contig \\
\hline MtABCG1 & TC131744 & IMGA|AC171532_22.4 & AC171532_22 & 8,343 & 4,866 & 1,469 & 4 & mth2-5a15 (chr07_pseudomolecule_IMGAG_V2) \\
\hline MtABCG2 & & IGA|AC171532_32.4 & C171532_32 & 028 & & & 4 & $7^{-1}$ nseudon \\
\hline MtABCG3 & TC123185 & IMGA|AC171532_16.4 & AC171532_16 & 8,014 & 4,510 & 1,462 & 4 & mth2-5a15 (chr07_pseudomolecule_IMGAG_V2) \\
\hline MtABCG4 ${ }^{\mathrm{b}}$ & $\ldots$ & IMGA|AC147517_9 & AC147517_9 & 8,285 & 4,329 & 1,443 & 4 & chr07_pseudomolecule_IMGAG \\
\hline MtABCG5 & $\ldots$ & IMGA|AC171532_21.4 & AC171532_21 & 7,120 & 4,413 & 1,471 & 4 & mth2-5a15 (chr07_pseudomolecule_IMGAG_V2) \\
\hline MtABCG6 & & IMGA|AC171532_26.4 & AC171532_26 & 7,089 & 4,575 & 1,433 & 4 & a15 (chr07_pseudomolecule_IMGAG_V2) \\
\hline MtABCG7 & TC136665 & IMGA|AC14 & $\mathrm{AC} 1446$ & 8,440 & 4,494 & & 4 & i_V2) \\
\hline MtABCG8 & TC114314 & IMGA|AC144617_23.5 & AC144617_23 & 10,538 & 5,649 & & 4 & mth2-7m9 (chr01_pseudomolecule_IMGAG_V2) \\
\hline MtABCG9 & TC107192 & IMGA|AC144340_29.4 & AC144340_29 & 7,261 & 4,479 & 1,493 & 4 & mth2-7k2 (chr02_pseudomolecule_IMGAG_V2) \\
\hline MtABCG10 & TC107191 & IMGA|AC144340_22 & AC144340_22 & 7,078 & 4,527 & 1,429 & 4 & chr02_pseudomolecule_IMGAG \\
\hline MtABCG11 & $\ldots$ & )_9.4 & & 7,820 & 5,631 & & 4 & 2_pseudon \\
\hline MtABCG12 & & IMGA|AC17 & AC170988_18 & 7,406 & 4,284 & 1,428 & 4 & 2 (chr07_pseudomolecule_IMGĀG_V2) \\
\hline MtABCG13 & & IMGA|AC170988_6.5 & AC170988_6 & 7,320 & 4,368 & 1,456 & 4 & mth2-53m12 (chr07_pseudomolecule_IMGAG_V2) \\
\hline MtABCG14 & & IMGA|AC170988_17.5 & AC170988_17 & 9,304 & 4,677 & 1,473 & 4 & mth2-53m12 (chr07_pseudomolecule_IMGAG_V2) \\
\hline MtABCG15 & TC102393 & IMGA|AC174352_33.5 & AC174352_33 & 8,260 & 4,522 & 1,429 & 4 & mth2-7o1 (chr08_pseudomolecule_IMGAG_V2) \\
\hline & TC107302 & & & 10,684 & & & 4 & mth2-30j23 (chr08_pseudomolecule_IMGAG_V2) \\
\hline MtABCG17 & & IMGA|CR954192_34.5 & CR954192_34 & 8,686 & 3,738 & 1,246 & 3 & mth2-15h3 (chr05_pseudomolecule_IMGAG_V2) \\
\hline MtABCG18 & $\begin{array}{l}\text { TC97256, } \\
\text { TC104271, }\end{array}$ & & & & & & & \\
\hline MtABCG19 & $\ldots$ & IMGA|AC202577_4.4 & AC202577_4 & 6,878 & 3,558 & 1,115 & 3 & mth2-176a4 (chr08_pseudomolecule_IMGAG_V2) \\
\hline
\end{tabular}

\footnotetext{
${ }^{a}$ Pleitropic drug-resistant (PDR) signatures.

${ }^{\mathrm{b}}$ Reference from Mt 1.0 database (Medicago Genome Sequencing Consortium 2006).
} 
ent $M$. truncatula organs. A vast majority of ABCG genes were expressed in the 6-week-old plants (Fig. 2A). Only four transcripts (MtABCG2, MtABCG5, MtABCG6, and MtABCG12) were not detected. For them, a new set of primers was designed and tested but, again, no product was detected (not shown), indicating that these genes were most likely not transcribed in the tested condition. The latter hypothesis is supported by the fact that no EST of the corresponding transcripts were found in the available databases. We have observed a high number of genes expressed in roots (14 of 19); namely, $M t A B C G 1, M t A B C G 3$ to MtABCG4, MtABCG7 to MtABCG11, and $M t A B C G 14$ to $M t A B C G 19$ (Fig. 2A). In contrast to roots, only eight genes were expressed in leaves (MtABCG8 to
$M t A B C G 11, M t A B C G 15$ to $M t A B C G 17$, and $M t A B C G 19)$. In addition, their mRNAs accumulated to relatively low levels (Fig. 2A). Compared with leaves, a higher number of different full-size MtABCG mRNAs was present in flowers $(n=11)$ and stems $(n=9)$. Western blot analyses involving ABCG subfamily-specific antibodies obtained against conserved PDR motifs (Ducos et al. 2005) were consistent with RT-PCR data. They indicated the highest accumulation of MtABCG proteins in roots and flowers (Fig. 2B). Interestingly, some genes showed organ-specific expression. MtABCG3 and MtABCG4 were found only in roots. The latter belong to a highly conserved group clustering within a clearly separated clade, MtABCG1 to MtABCG4. Moreover, mRNAs of MtABCG13 and

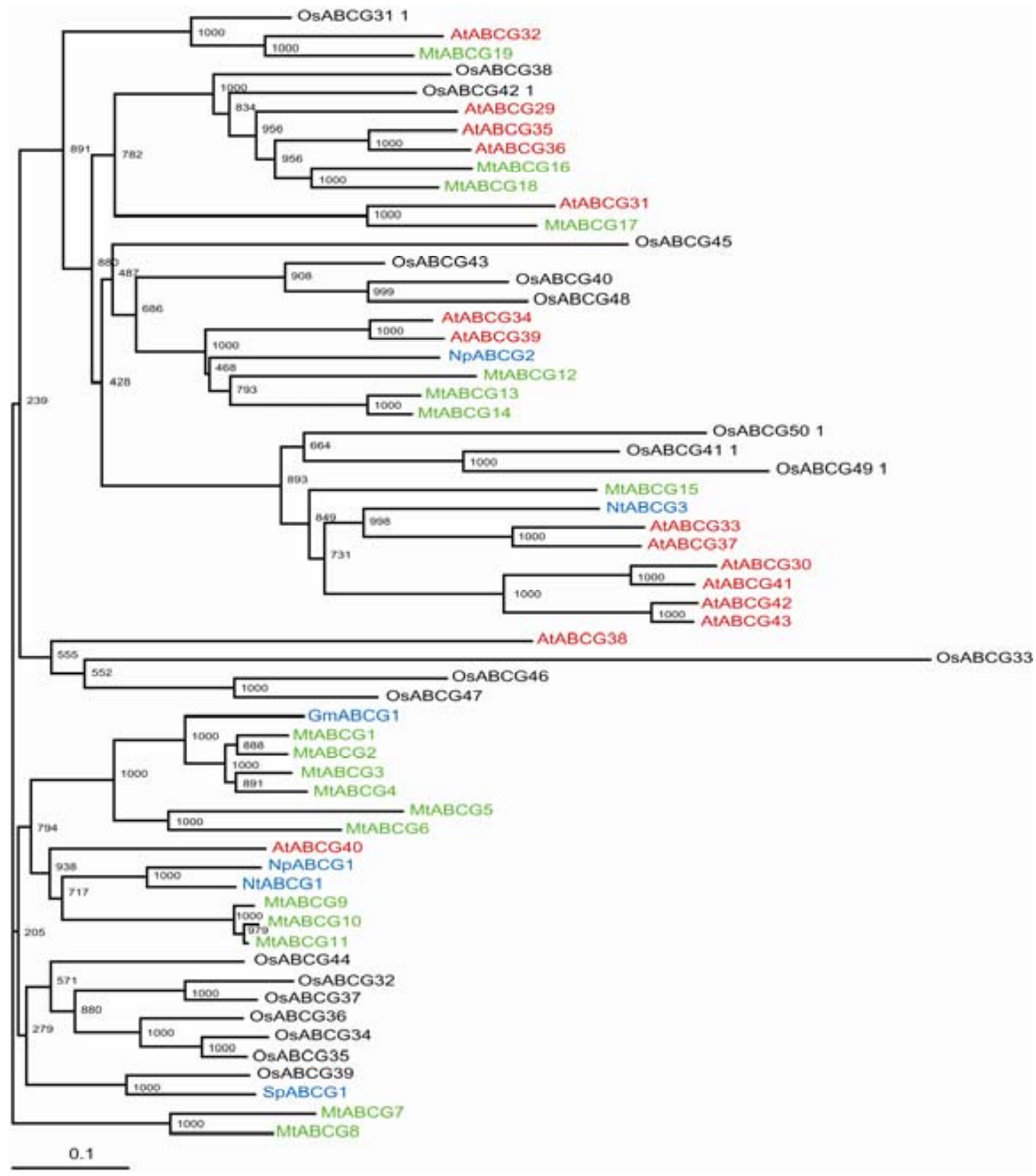

Fig. 1. Phylogenetic analysis of plant full-size ABCG proteins from: Medicago truncatula (Mt), Arabidopsis thaliana (At), Oryza sativa (Os), Nicotiana plumbaginifolia $(\mathrm{Np})$, N. tabacum $(\mathrm{Nt})$, Spirodela polyrrhiza $(\mathrm{Sp})$, and Glycine max $(\mathrm{Gm})$. The full-length protein sequences were aligned using a Clustal W program (Thompson et al. 1994) and the neighbor-joining method (PHYLIP package) (Felsenstein 1985) was used to construct the phylogenetic tree with 1,000 bootstrap replicates. The protein names have been given according to the recently proposed unified nomenclature (Verrier et al. 2008). A. thaliana and $O$. sativa accession numbers are from Verrier and associates (2008). The numbers for S. polyrrhiza (SpABCG1) (Smart and Fleming 1996), G. max (GmABCG1) (Eichhorn et al. 2006), N. plumbaginifolia (NpABCG1 [Jasinski et al. 2001] and NpABCG2 [Trombik et al. 2008]), and N. tabacum (NtABCG1 [Sasabe et al. 2002] and NtABCG3 [Ducos et al. 2005]) were published earlier. The ABCG from M. truncatula are in green, A. thaliana in red, O. sativa in black, and others in blue. 
MtABCG14 which encode transporters sharing a high percentage of amino acid identity $(91 \%)$ specifically accumulated in two different organs, flowers and roots, respectively (Fig. 2A).

\section{Full-size ABCG gene expression in $M$. truncatula roots.}

Especially high abundance of the MtABCG gene products in roots prompted us to more detailed studies of their expression in this organ. At first, we used roots of plants grown in sterile conditions. The roots were dissected into three arbitrarily chosen zones-zone 1 ( 0 to $0.5 \mathrm{~cm}$ form a root tip) followed by zone $2(0.5$ to $2 \mathrm{~cm})$ and zone $3(2$ to $4 \mathrm{~cm})$ - and the accumulation of MtABCG mRNAs was analyzed by RT-PCR. We observed that transcripts of at least three genes (MtABCG3, $M t A B C G 8$, and $M t A B C G 14$ ) were especially abundant in the region close to a root tip (zone 1). The rest of the MtABCG mRNAs were detected along the entire root, with the exception of $M t A B C G 4$, found exclusively in zone 2, and $M t A B C G 19$, found in zone 3 (Fig. 3A).

Symbiosis of the legume plants and prokaryotes known as rhizobia (Rhizobium and Sinorhizobium spp.) is a particular

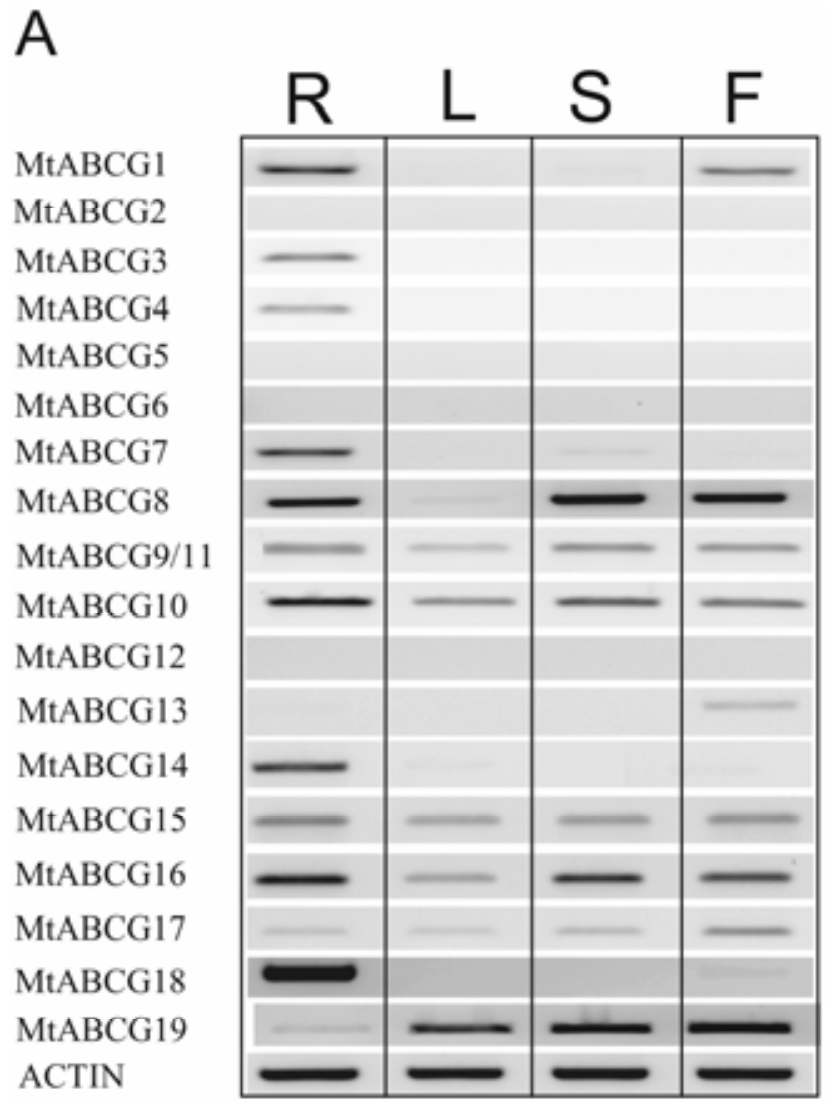

B

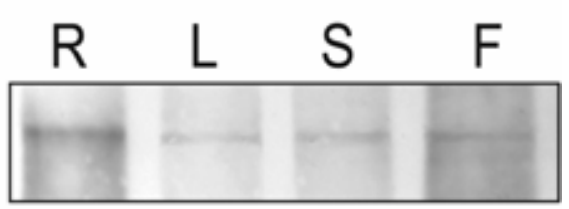

Fig. 2. Reverse-transcriptase polymerase chain reaction (RT-PCR) analysis of the full-size MtABCG gene expression in different organs. A, cDNAs were synthesized from total RNA isolated from roots (R), leaves (L), stems (S), and flowers (F) and subjected to RT-PCR (30 cycles). As a reference, the expression of actin was determined. B, Western blot analysis of the MtABCG proteins in microsomal fractions $(30 \mu \mathrm{g})$ extracted from roots $(\mathrm{R})$, leaves $(\mathrm{L})$, stems $(\mathrm{S})$, and flowers $(\mathrm{F})$. example of a plant-microbe interaction, which results in the formation of a new organ known as a root nodule. The symbiotic nodules are a place of intense trade in which ABC transporters can be involved. Proteomic studies of nodule bacteria led to the identification of several ABC transporters, certain of them being necessary for the exchange of metabolites (Dupont et al. 2004). Considering the above observation, we attempted to determine MtABCG gene expression in nodules forming on seedling roots upon a Sinorhizobium meliloti infection (Fig. 3B). Undertaken RT-PCR analysis showed a particularly high level of MtABCG16 mRNA accumulation. It was maintained up to 21 days after inoculation and than decreased (Fig. 3B). Interestingly, other MtABCG transcripts accumulated in nodules to visibly lower levels than in noninoculated roots (Fig. 3). Observation concerning MtABCG16 is consistent with the DNA microarray data obtained for a corresponding EST (TC107302) during symbiotic nodules analysis (Benedito et al. 2008).

To determine whether $M t A B C G$ are up- or downregulated during symbiotic interactions, the pattern of their expression was also examined in seedling roots after inoculation with $S$. meliloti. For the majority of the genes, there were no significant differences in the level of MtABCG mRNA accumulation compared with noninfected control plants at the arbitrarily chosen time points (Fig. 4A). Several genes were expressed constitutively at a low level (MtABCG4, MtABCG13, $M t A B C G 17$, and $M t A B C G 19)$ and certain others were not expressed at all (MtABCG2, MtABCG5, MtABCG6, and $M t A B C G 12)$. As a result, only two genes belonging to different clades (Fig. 1)—MtABCG10 (appearing as upregulated) and MtABCG16 (showing high level of expression in nodules) were chosen for the further analysis by real-time quantitative (q)RT-PCR, which revealed only slight changes in the level of expression of both genes. However, they were statistically significant in the case of MtABCG10 at the early stage of infection (Fig. 4B).

\section{Changes in the full-size MtABCG expression upon fungal pathogen infection.}

The expression of several ABCG transporters was shown as induced by biotic stress; for example, NpPDR1 (NpABCG1) (Stukkens et al. 2005) and AtPDR12 (AtABCG40) (Campbell et al. 2003); and dysfunction of certain others was associated with an increased plant susceptibility to pathogenic fungal infections (Cruzet et al. 2006). Taking into account the above facts, we examined how the MtABCG gene expression is affected by infection with two common pathogenic fungi, Fusarium culmorum and Phoma medicaginis, both causing severe diseases of forage and grain legumes.

F. culmorum is a necrotrophic, soilborne pathogenic fungus, infecting root system and causing spring black stem and root rot (Tivoli at al. 2006). The infection of $M$. truncatula roots with $F$. culmorum resulted in an increased transcript accumulation, especially that of MtABCG10 and MtABCG16 (Fig. 5). It is worth noting that MtABCG10 shares $70 \%$ amino acid identity with AtPDR12, expression of which has been induced in Arabidopsis upon fungal pathogen infection, including Fusarium spp. (Campbell et al. 2003). MtABCG16 is a close homolog (72\% amino acid identity) of AtPDR8/PEN3 (AtABCG36) (Kim et al. 2007; Stein et al. 2006) which, in Arabidopsis, contributes to defense against pathogenic fungi such as Erysiphe pisi and Phytophthora infestans (Stein et al. 2006).

A similar tendency was observed for both genes (MtABCG10 and MtABCG16) when we analyzed the material derived from the $M$. truncatula leaves infected with the hostspecific fungus Phoma medicaginis (Fig. 6). The genus Phoma 
is a necrotrophic pathogen responsible for frequently occurring diseases of legumes, such as leaf spot (Kamphuis et al. 2008). Although, in general, a low number of different $M t A B C G$ mRNAs were present in leaves, the detected ones showed a clear response to $P$. medicaginis (Fig. 6). MtABCG10 upregulation is in agreement with DNA microarray data obtained for corresponding EST (TC107191 and MtD00930_GC) (Journet et al. 2002).

In general, the analysis of MtABCG mRNAs accumulation presented above suggests that symbiotic and pathogenic interactions especially affect two genes: MtABCG10 and $M t A B C G 16$ (Figs. 4A, 5A, and 6A). Their expression is particularly elevated in leaves inoculated with $P$. medicaginis (approximately fivefold increase of mRNA accumulation) (Fig. 6B). A less substantial although statistically significant increase of MtABCG10 and MtABCG16 mRNA accumulation is observed in S. meliloti- or F. culmorum-inoculated roots (Figs. 4B and $5 \mathrm{~B}$ ). Based on a Western blot analysis involving ABCG subfamily-specific antibodies, one can conclude that the upregulation of ABCG genes is also observed at the protein level (Supplementary Fig. S2).

\section{DISCUSSION}

Sequencing of several eukaryotic genomes revealed a significantly higher number of putative $\mathrm{ABC}$ transporter genes in plants $(>120)$ than in other multicellular organisms (approximately 50) (Jasinski et al. 2003; Sanchez Fernandez et al. 2001; Sugiyama et al. 2006). Although this fact suggests that $\mathrm{ABC}$ proteins are of special importance for plants, our knowledge about their functioning is still very poor. To better recognize this problem, we attempted to characterize full-size ABCG transporter genes from $M$. truncatula, a model legume plant. We focused on the ABCG subfamily because some of its members were shown to be implicated in plant-microbe inter- actions (Campbell et al. 2003; Kobae et al. 2006; Stein et al. 2006; Stukkens et al. 2005). Out of all M. truncatula genes we analyzed, 19 were classified as members of this subfamily. The number of the identified ABCG genes was higher than in Arabidopsis and close to that of rice (Cruzet et al. 2006). In addition, we found, in various $M$. truncatula cDNA resources, only one EST (TC 102516) without a corresponding full-length genomic sequence present in the Mt 2.0 database. TC 102516 seems to be a homolog of MtABCG10, sharing $82 \%$ nucleotide identity. Unfortunately, we did not know the whole coding sequence of this gene; thus, the product of its expression was not included in the phylogenetic tree (Fig. 1). Altogether, these suggest that at least 20 full-size MtABCG genes are present in the $M$. truncatula genome and that the majority of them have been included in our studies.

Sugiyama and coworkers have made a similar attempt to inventory $\mathrm{ABC}$ transporter genes in L. japonicus (Sugiyama et al. 2006). Among 91 identified genes, only 12 have been classified as coding for full-size ABCG proteins. The applied methodology (domain-based clustering analysis) is most likely the reason why a relatively low number of full-size ABCG genes $(L j A B C G)$ has been found in Lotus spp. (for $70 \%$ of the genome coverage). The genome-wide screening, based exclusively on two domains (TMD and NBD), could make the identification of close homologs difficult. MtABCG9 to $M t A B C G 11$ well exemplify the situation. The overall identity of their nucleotide sequence is more than $90 \%$ and only full sequence alignment enabled us to distinguish these three genes.

Closer analysis of $12 \mathrm{LjABCG}$ revealed that at least 5 of them are homologs of Arabidopsis AtPDR12 (AtABCG40). Interestingly, we have also identified three transporters (MtABCG9 to MtABCG11) having 70\% amino acid identity with AtABCG40 and four more (MtABCG1 to MtABCG4) for which identity to the latter protein is greater than $65 \%$. The
A

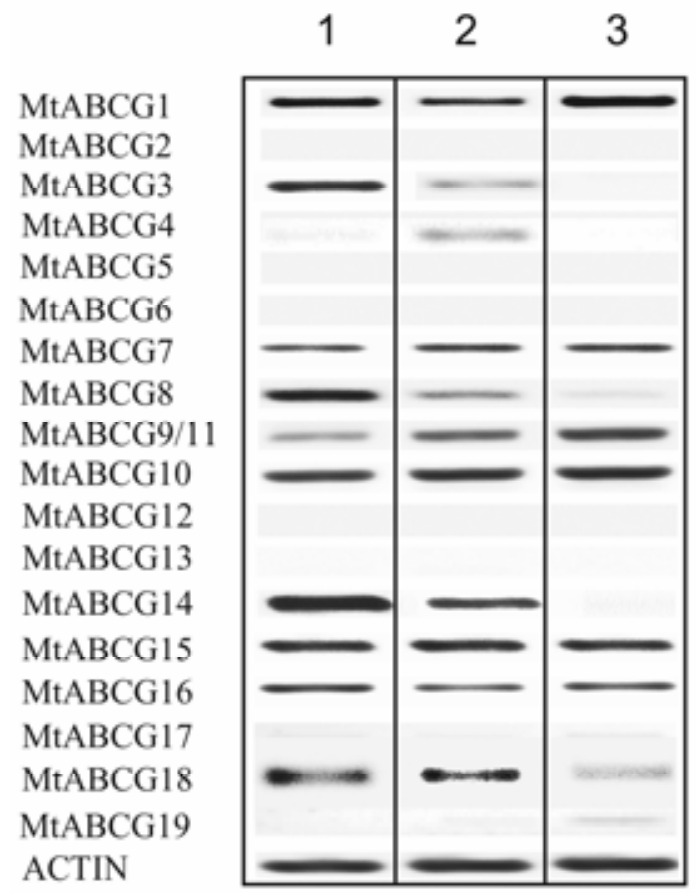

B

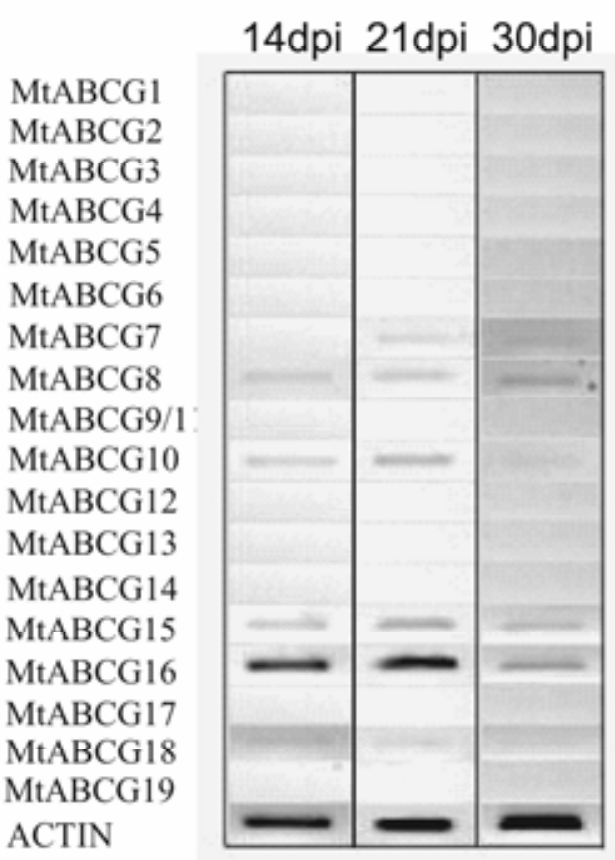

Fig. 3. Reverse-transcriptase polymerase chain reaction analysis (30 cycles) of the MtABCG gene expression in roots. A, MtABCG mRNA accumulation in the arbitrary selected root segments of the 7-day-old Medicago truncatula seedlings: zone 1 (0 to $0.5 \mathrm{~cm})$ from a root tip followed by zone 2 ( 0.5 to $2 \mathrm{~cm})$ and zone 3 ( 2 to $4 \mathrm{~cm})$. B, MtABCG mRNA accumulation in the root nodules. The cDNAs were synthesized using total RNA isolated from nodules collected 14,21 , and 30 days postinoculation (dpi). The actin gene transcript was analyzed as an internal control. 
above-mentioned TC 102516 also displays a high similarity to $M t A B C G 10$. Accordingly, one can state that at least eight homologs of AtABCG40 are encoded in the M. truncatula genome. It seems that such a redundancy of $A t A B C G 40$-like genes may be a unique feature of legume genomes because it is observed in both M. truncatula and Lotus spp. but not in rice.

An RT-PCR analysis showed that, in the 6-week-old $M$. truncatula plants, only seven $M t A B C G$ genes were expressed in all examined organs. The mRNAs of $14 M t A B C G$ genes were detected in root tissues. Among them there were several with an especially high level of accumulation in roots (MtABCG3, MtABCG4, MtABC 7, MtABCG14, and $M t A B C G 18)$, root nodules (MtABCG16), or in particular root zones (MtABCG3, MtABCG4, and MtABCG19). The number of $M t A B C G$ expressed in other organs was markedly smaller. Similar observation was made by Sugiyama and coworkers.
Six of eight $L j A B C G$ they examined also have a higher transcript level in roots than in other organs (Sugiyama et al. 2006). In addition, they observed the increased accumulation of six LjABCG transcripts (out of eight examined) in roots 3 weeks after inoculation with Mesorhizobium loti. We have also noted some changes in MtABCG expression in Medicago truncatula roots during symbiotic interactions with $S$. meliloti. However, it is difficult to compare Sugiyama's data concerning the whole roots with ours obtained for seedling roots (representing the early stage of infection) and fully developed nodules. Nevertheless, altogether, they indicate that LjABCG and MtABCG can be involved in symbiosis between legumes and rhizobia.

As yet, little is known about the function of the plant $\mathrm{ABC}$ transporters in roots. The rhizosphere is a place of intense interactions with bacteria, fungi, insects, and other plants.
A

MtABCG1
MtABCG2
MtABCG3
MtABCG4
MtABCG5
MtABCG6
MtABCG7
MtABCG8
MtABCG9/1
MtABCG10
MtABCG12
MtABCG13
MtABCG14
MtABCG15
MtABCG16
MtABCG17
MtABCG18
MtABCG19
ACTIN

B

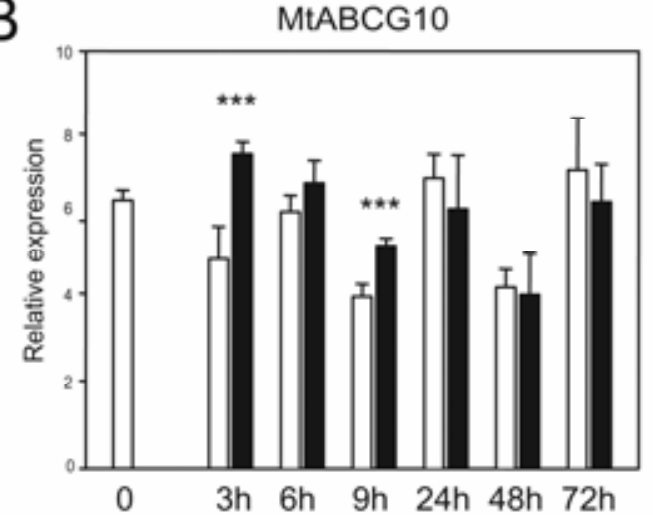

Control 0
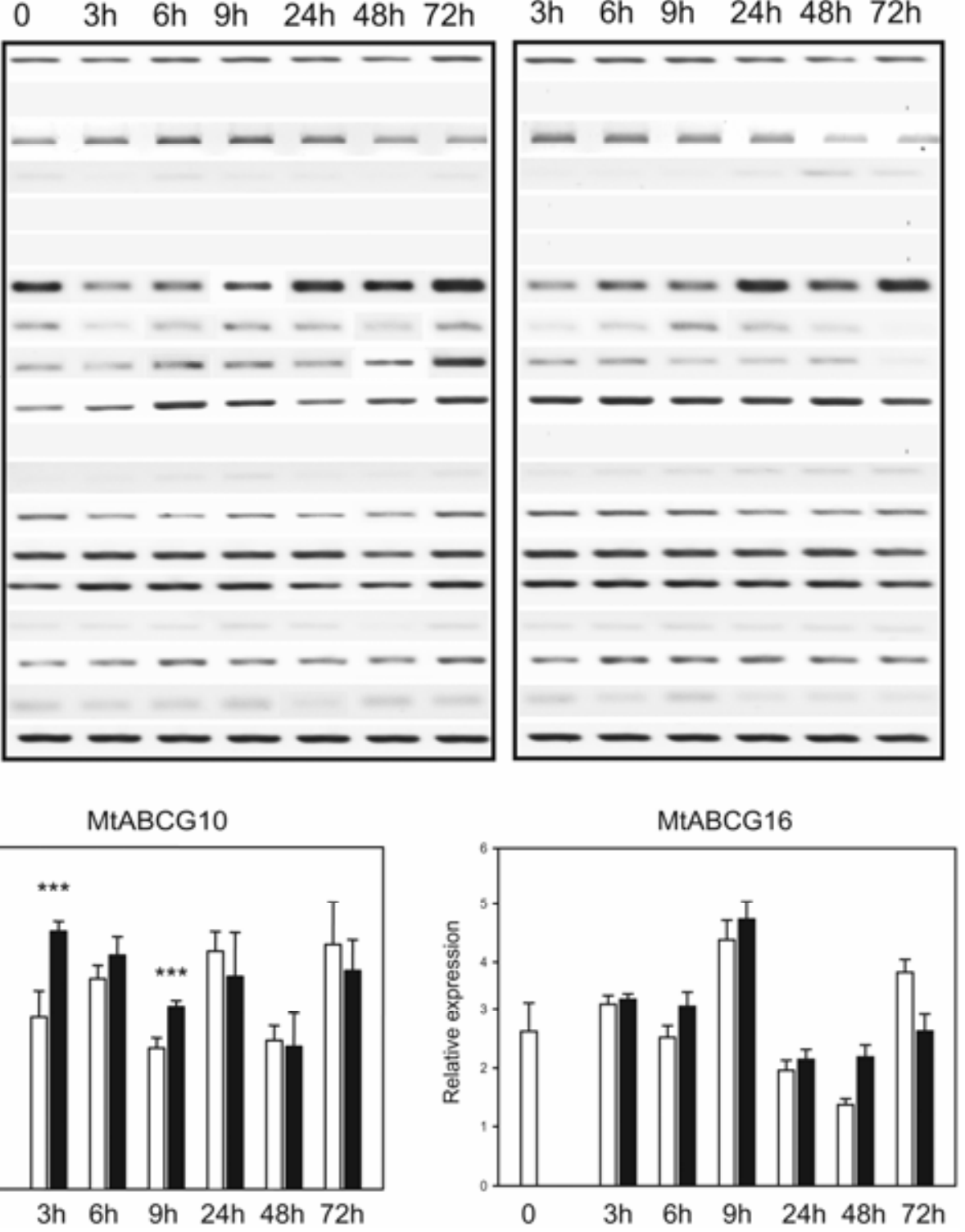

Fig. 4. Expression of the full-size MtABCG genes in roots upon Sinorhizobium meliloti infection. A, Reverse-transcriptase polymerase chain reaction (RTPCR) analysis (27 cycles) of the MtABCG mRNA accumulation in noninfected (control) and infected seedlings roots at the indicated time points. The actin gene transcript was used as an internal control. B, Quantitative (q)RT-PCR analysis of the MtABCG10 and MtABCG16 gene expression. Transcript levels are shown as values relative to those of actin used as the internal control. White bars $=$ noninfected and black bars $=$ infected material. Data represent the means \pm standard deviation of two independent biological experiments. Each experiment involved five plants per condition and four technical replicates of qRTPCR. Significant differences from the control plants determined by Student's $t$ test are indicated $(*, * *$, and $* * *=P<0.05,0.02$, and 0.01 , respectively). 
Thus, an efficient transport through cellular membranes is necessary to ensure effective flow of different molecules (e.g., root exudates with defense or signaling properties). In the rhizome of Coptis japonica, one member of the ABCB subfamily was recognized as an importer of alkaloids (Shitan et al. 2003). Other ABCG from Arabidopsis, namely PGP1 (AtABCB1) and PGP4 (AtABCB4), were shown to be particularly expressed in the roots and root tip, where they contribute to distribution of auxin (Geisler et al. 2005; Santelia et al. 2005; Terasaka et al. 2005). To date, ABCG proteins have not been associated with auxin transport. However, Arabidopsis PDR9 (AtABCG37) has been shown to be capable of transporting synthetic auxin 2,4-D.
It seems that the AtPDR9 can specifically efflux 2,4-D out of plant cells without affecting endogenous auxin transport (Ito and Grey 2006). Because of the high expression in the lateral root cap and epidermis, it was suggested that AtPDR9 can also participate in communication between plant roots and other organisms (Ito and Grey 2006). In addition, the involvement of two other Arabidopsis ABCG proteins, AtPDR6 (AtABCG34) and AtPDR7 (AtABCG35), in the metabolite secretion was postulated, based on the observation that the deletion of their genes affected the composition of root exudates (Badri et al. 2008).

Infection of $M$. truncatula roots with $F$. culmorum resulted in the slightly higher expression of MtABCG10 and
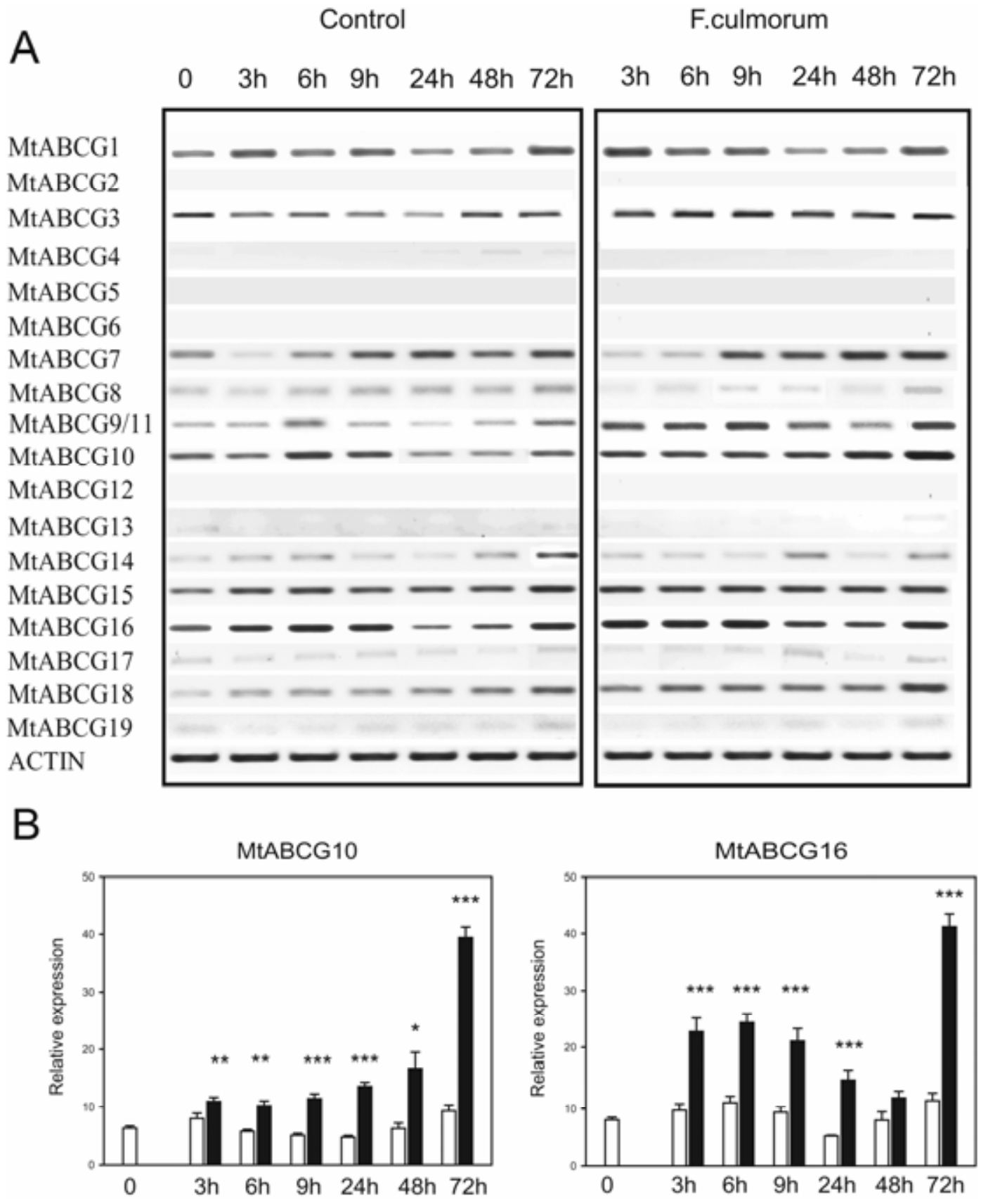

Fig. 5. Expression of the full-size MtABCG genes in roots upon Fusarium culmorum infection. A, Total RNA was isolated at the indicated time points from noninfected (control) and infected seedlings roots. cDNAs obtained after a reverse transcription were subjected to polymerase chain reaction (PCR) analysis (27cycles). The actin gene transcript was used as an internal control. B, Quantitative reverse-transcriptase (qRT)-PCR analysis of MtABCG10 and $M t A B C G 16$ gene expression in untreated (white bars) and infected (black bars) material. Transcript levels are shown as values relative to those of actin used as the internal control. Data represent the means \pm standard deviation of two independent biological experiments. Each experiment involved five plants per condition and four technical replicates of qRT-PCR. Significant differences from the control plants determined by Student's $t$ test are indicated (*, **, and $* * *=P<0.05,0.02$, and 0.01 , respectively). 
$M t A B C G 16$ than during symbiotic interactions with bacteria. However, both genes were much more strongly induced by $P$. medicaginis in leaves, thus suggesting a relation between their activation and fungal infections. Accordingly, one can hypothesize that ABCG proteins are involved not only in plant-symbiont but also in plant-fungus interactions in M. truncatula. .

In addition, the collected data indicate that both transcripts (MtABCG10 and MtABCG16) are under the influence of the diurnal cycle, showing a relatively low level of accumulation at the early hours of the light period (Fig. 6B, T0 = 10:00 a.m.).

Numerous MtABCG mRNAs were found in M. truncatula flowers (Fig. 2). One of them (MtABCG13) was detected exclusively in this organ. Its close homologs are NpPDR2
(NpABCG2) from N. plumbaginifolia and AtPDR11 (AtABCG39) from Arabidopsis, both displaying 70\% amino acid identity. Expression of the NpPDR2 gene was shown to be dependent on pollination and its transcript was found only in pollinated style. No specific phenotype was observed in plants silenced for NpPDR2 (Trombik et al. 2008). The AtPDR 11 was also shown as flower-specific (carpel and ovary) (Zimmermann et al. 2004). The full-size ABCG proteins were proposed to transport floral fragrances in flowers (Stukkens et al. 2005), signaling molecules or hormones induced by processes such as pollination (Trombik et al. 2008). Surprisingly, we found that $M t A B C G 14$, a close homolog of $M t A B C G 13$, is exclusively expressed in roots. This suggests that either both

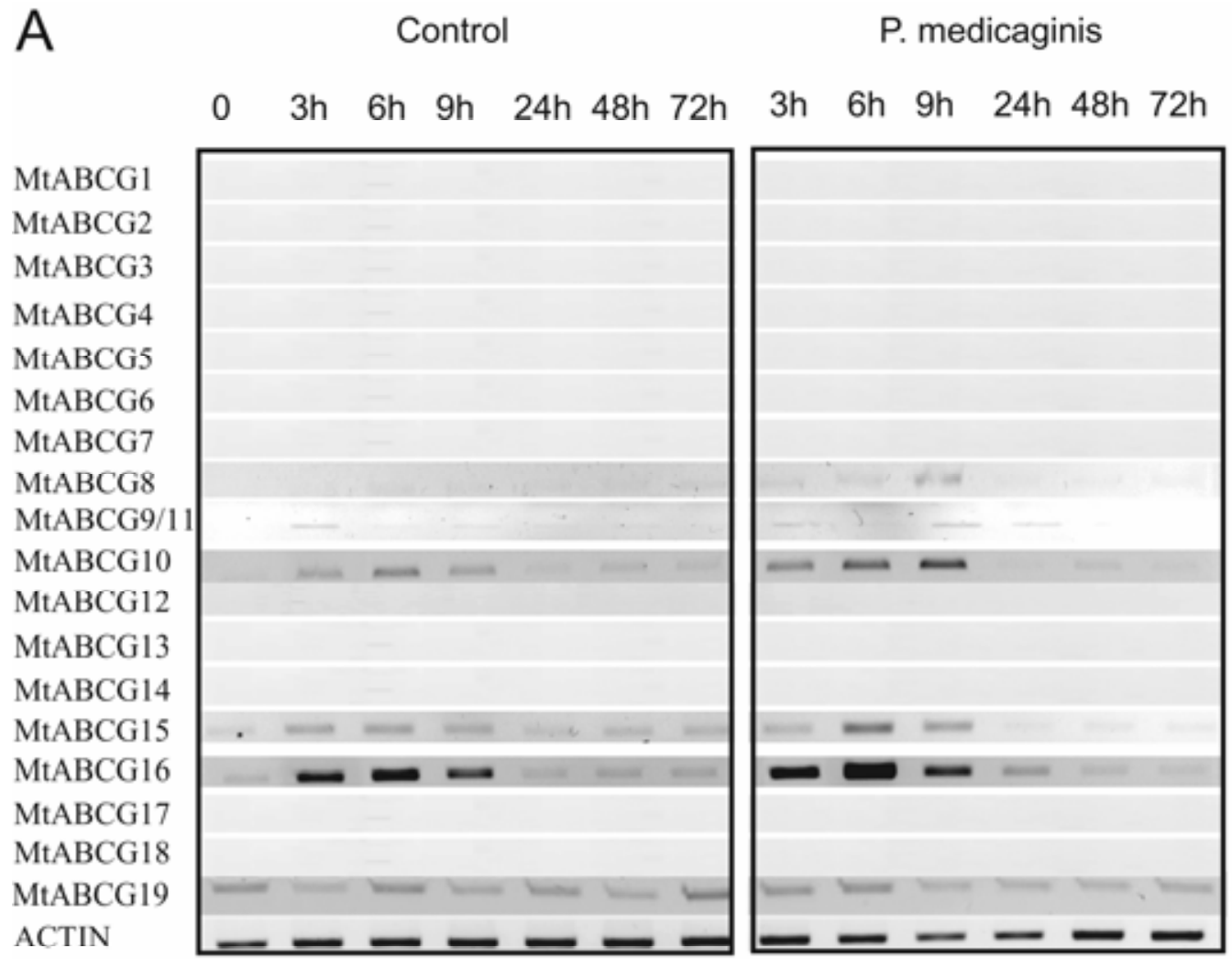

B
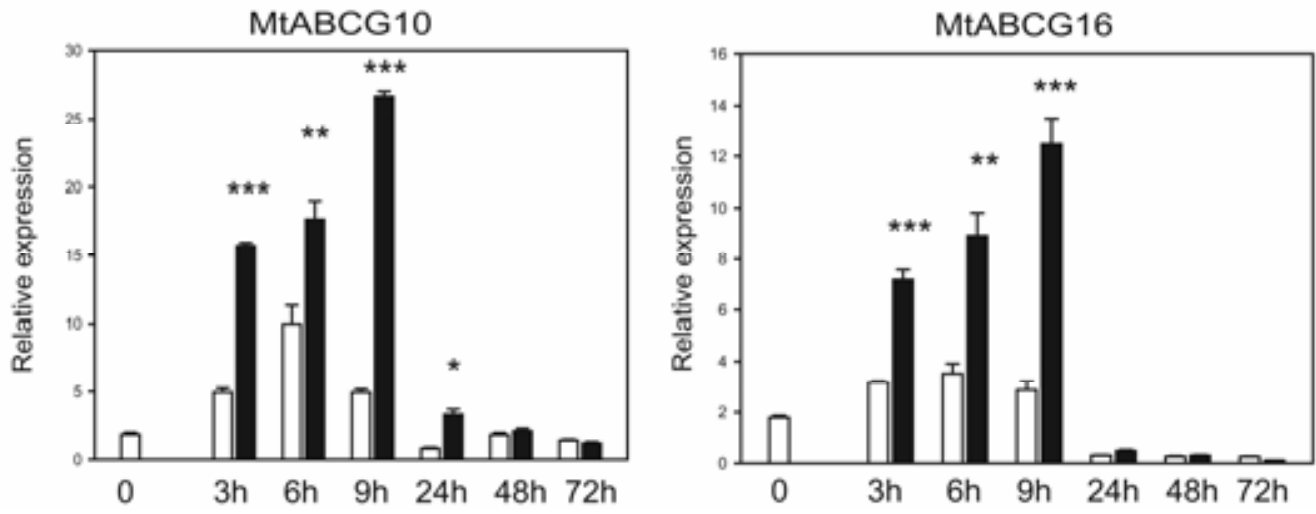

Fig. 6. Expression of the full-size MtABCG genes in leaves upon Phoma medicaginis infection. A, Total RNA was isolated at the indicated time points from noninfected (control) and infected leaves. cDNAs obtained after reverse transcription were subjected to polymerase chain reaction (PCR) analysis (25 cycles). The actin gene transcript was used as an internal control. B, Quantitative reverse-transcriptase (qRT)-PCR analysis of $M t A B C G 10$ and $M t A B C G 16$ gene expression in untreated (white bars) and infected (black bars) leaves. Transcript levels are shown as values relative to those of actin used as the internal control. Data represent the means \pm standard deviation of two independent biological experiments, five plants per condition, and four technical replicates of qRT-PCR. Significant differences from the control plants determined by Student's $t$ test are indicated $(*, * *$, and $* * *=P<0.05,0.02$, and 0.01 , respectively). 
fulfill similar roles but in different organs or they have different substrates.

Identification of the substrates for the ABCG proteins still remains an essential and unsolved issue. Members of the ABCG subfamily are capable of transporting a wide range of unrelated molecules. In addition, as shown, for instance, for the Arabidopsis mutants Atpdr6 and Atpdr7, one compound may be a substrate for different transporters, indicating their functional redundancy (Badri et al. 2008). On the other hand, one ABCG protein can transport different molecules; for example, Arabidopsis PDR8 (AtABCG36) (close homolog of MtABCG16) both contributes to defense against pathogenic fungi (probably by transporting chemicals that mediate pathogen resistance) and functions as an efflux pump for cadmium or cadmium conjugates (Kim et al. 2007; Kobae et al. 2006; Stein et al. 2006). Therefore, a high level of MtABCG16 mRNA in leaves and nodules can have different biological meanings. The expression of several MtABCG transporters in different organs such as stems, leaves, and flowers (e.g., $M t A B C G 10$ and $M t A B C G 15$ to $M t A B C G 17)$ also suggests their possible functional diversity.

The ABC transporters have been shown to be active in transport of various natural products, including plant secondary metabolites. Although, currently, nothing is known about the MtABCG substrates, it should be noted that, during $P$. medicaginis infection, a higher accumulation of medicarpin (isoflavonoid phytoalexin) and its precursors (e.g., formononetin glucoside) was observed in M. truncatula leaves (Deavours and Dixon 2005). The medicarpin is a possible substrate for the ABC transporters, as shown in biochemical assays with vacuolar membrane vesicles of mung bean (Vigna radiata) (Li et al. 1997). Vacuolar uptake experiments indicated that, depending on the plant species and type of conjugated moiety (glucoside or glucuronide), different transporters (either directly energized ABC-type transporters or secondarily energized antiporters) are involved in the trafficking of phenolic compounds (Yazaki 2005). Molecular identity of such transporters starts to be recognized (Goodman et al. 2004; Marinova et al. 2007). To date, most of the characterized ABCG transporters have been localized in plasma membrane (Ito and Gray 2006; Jasinski et al. 2001; Kobae et al. 2006). However, one cannot exclude the possibility that they are also anchored in the other types of cellular membranes or membrane vesicles; participating, for instance, in compartmentation or turnover of secondary metabolites or their precursors. In soybean, the translocation of flavonoid compounds through the plasma membrane was shown to be fulfilled by $\mathrm{ABC}$ transporters most likely being members of the $\mathrm{ABCG}$ subfamily (Sugiyama et al. 2007). However, also in this case, identification of the concrete transporter or transporters is still necessary.

Apart from phenolic compounds, terpenoids also appeared as possible substrates for the ABCG transporters (e.g., NpPDR1 and diterpene sclareol) (Jasinski et al. 2001). In legumes, a group of sesqiterpene lactones have been identified as a root-released signal inducing the hyphal branching of mycorrhizal fungi (Akiyama et al. 2005). Secretion of such terpenoid signaling molecules can be a function of certain ABCG transporters (Cruzet et al. 2006). Analysis of EST data from the electronic northern recourses revealed that certain MtABCG transporters (e.g., MtABCG16 and MtC00386_GC) are clearly upregulated after Glomus spp. infection. However, it must be emphasized that caution is needed in the prediction of homologous gene functions in different species. A recent comparative transcriptome analysis of $M$. truncatula versus Arabidopsis revealed a significant divergence in the developmental expression profiles of highly similar genes, which indi- cates that a phylogenetic analysis alone is insufficient for the elucidation of their possible function (Benedito et al. 2008). Identification of transporters and their putative substrates is essential for better understanding interactions of legume plants with other organisms. The data presented here represent a scaffold for further detailed studies of physiological functions of particular MtABCG proteins and their role in the transport of various molecules throughout plant tissue.

\section{MATERIALS AND METHODS}

\section{Plant material.}

Plants (M. truncatula Gaertn. ecotype Jemalong J5, provided by A. Kondorosi, CNRS, Gif-sur-Yvette, France) were grown in pots containing a mixture of sand and perlite (1:1) under controlled greenhouse conditions, with an average temperature of $25^{\circ} \mathrm{C}, 50 \%$ humidity, and a 16 -h photoperiod. Plants were watered every 2 days and supplemented once per week with N-P-K fertilizer (6:3:6).

\section{P. medicaginis infection of $M$. truncatula leaves.}

Six-week-old plants were inoculated with $P$. medicaginis. To this end, conidia of $P$. medicaginis Malbr. et Roum. (strain PH 33, from the collection of the Institute of Plant Genetics, Polish Academy of Sciences) were plated on potato dextrose agar and incubated at $28^{\circ} \mathrm{C}$ for 3 weeks until the appearance of pycnidia. Pycnidiospores were collected by flooding the plate with sterile $0.1 \%$ (vol/vol) Tween 20 . Next, the suspension was filtered to remove parts of mycelia, and spore concentration was determined by counting. Routinely, an inoculation mixture containing $2 \times 10^{6}$ spores $/ \mathrm{ml}$ was sprayed on the plants. Leaves in control and infected plants were collected 3, 6, 9, 24,48 , and $72 \mathrm{~h}$ after inoculation and immediately frozen in liquid nitrogen.

\section{S. meliloti or $F$. culmorum infection of M. truncatula seedlings.}

$M$. truncatula seedlings were germinated on a water-saturated Whatman disks in petri plates and kept in a greenhouse as described above. Seven-day-old seedlings were inoculated with $S$. meliloti Rm1021 strain (approximately $2 \times 10^{6}$ cells per milliliter in $10 \mathrm{mM} \mathrm{MgSO}$, $0.2 \mathrm{ml}$ per root) (Meade et al. 1982) or F. culmorum strain Cul-3 from the collection of the Institute of Plant Genetics, Polish Academy of Sciences $\left(5 \times 10^{6}\right.$ spores per milliliter in sterile water, $0.5 \mathrm{ml}$ per root). Seedling roots in control and infected plants were collected 3, 6, 9, 24, 48, and $72 \mathrm{~h}$ after the inoculation and immediately frozen in liquid nitrogen.

\section{Semiquantitative RT-PCR and real-time qRT-PCR analysis.}

RNA was isolated with the RNeasy plant RNA extraction kit (Qiagen). Genomic DNA was removed by on-column DNase (Qiagen) treatment. RNA samples used for qRT-PCR were additionally treated with DNA-free DNase (Ambion). Total RNA (500 ng) was converted to cDNA with Omniscript reverse transcriptase (Qiagen) according to the manufacture's protocol. PCR reactions were performed in an MJ Mini personal thermocycler (Bio-Rad) using 30, 27, or 25 cycles. qRTPCR analysis was performed in Rotor-Gene RG-3000 RealTime PCR machine (Corbett Research) using the SYBR Green PCR Master Mix (Applied Biosystems). To determine specificity of the primers used for qRT-PCR (single-product formation), melting curves were analyzed. Primers were accepted as specific if a single amplification product was detected. The threshold cycle method (Rasmussen 2001) was used to calculate mRNA concentration. The actin mRNA (TC107326) was used as a reference. 


\section{Microsomal fraction preparation and \\ Western blot analysis.}

The microsomal fractions were isolated from $300 \mathrm{mg}$ of plant material as described by Jasinski and associates (2001). Proteins (30 $\mu \mathrm{g}$ or, when mentioned, $40 \mu \mathrm{g}$ ) were separated by sodium dodecyl sulfate polyacrylamide gel electrophoresis (7\% polyacrylamide) and transferred to a polyvinylidene difluoride membrane (Millipore) by electroblotting (semidry transfer; Bio-Rad). The membrane was incubated with rabbit antibodies specific for the ABCG subfamily (GPDR) obtained against the conserved PDR motive (Ducos et al. 2005) and antibodies specific for $\mathrm{H}^{+}$-ATP-ase (W1G) (Morsomme et al. 1998).

\section{ACKNOWLEDGMENTS}

We thank S. Long for the Sinorhizobium meliloti strain, I. Femiak for excellent technical assistance, P. Kachlicki for help with Phoma medicaginis and Fusarium culmorum infections, M. Boutry for GPDR/W1G antibodies, and K. Brzezinski for help with the PHYLIP package. This work was supported by the Polish Ministry of Science and Higher Education grant N301 114 32/3928 and partially from the network "Genomics and transgenesis of crop plants" from the Polish Ministry of Science and Higher Education.

\section{LITERATURE CITED}

Akiyama, K., Matsuzaki, K., and Hayashi, H. 2005. Plant sesquiterpenes induce hyphal branching in arbuscular mycorrhizal fungi. Nature 435:824-827.

Badri, D. V., Loyola-Vargas, V. M., Broeckling, C. D., De-la-Peña, C., Jasinski, M., Santelia, D., Martinoia, E., Sumner, L. W., Banta, L. M., Stermitz, F., and Vivanco, J. M. 2008. Altered profile of secondary metabolites in the root exudates of Arabidopsis ATP-binding cassette transporter mutants. Plant Physiol. 146:762-771.

Benedito, V. A., Torres-Jerez, I., Murray, J. D., Andriankaja, A., Allen, S., Kakar, K., Wandrey, M., Verdier, J., Zuber, H., Ott, T., Moreau, S., Niebel, A., Frickey, T., Weiller, G., He, J., Dai, X., Zhao, P. X., Tang, Y., and Udvardi, M. K. 2008. A gene expression atlas of the model legume Medicago truncatula. Plant J. 55:504-513.

Campbell, E. J., Schenk, P. M., Kazan, K., Penninckx, I. A., Anderson, J. P., Maclean, D. J., Cammue, B. P., Ebert, P. R., and Manners, J. M. 2003. Pathogen-responsive expression of a putative ATP-binding cassette transporter gene conferring resistance to the diterpenoid sclareol is regulated by multiple defense signaling pathways in Arabidopsis. Plant Physiol. 133:1272-1284.

Crouzet, J., Trombik, T., Fraysse, A. S., and Boutry, M. 2006. Organization and function of the plant pleiotropic drug resistance ABC transporter family. FEBS (Fed. Eur. Biochem. Soc.) Lett. 580:1123-1130.

Deavours, B. E., and Dixon, R. A. 2005. Metabolic engineering of isoflavonoid biosynthesis in alfalfa. Plant Physiol. 138:2245-2259.

Ducos, E., Fraysse, S., and Boutry, M. 2005. NtPDR3, an iron-deficiency inducible ABC transporter in Nicotiana tabacum. FEBS (Fed. Eur. Biochem. Soc.) Lett. 579:6791-6795.

Dupont, L., Garcia, I., Poggi, M. C., Alloing, G., Mandon, K., and Le Rudulier, D. 2004. The Sinorhizobium meliloti ABC transporter Cho is highly specific for choline and expressed in bacteroids from Medicago sativa nodules. J. Bacteriol. 186:5988-5996.

Eichhorn, H., Klinghammer, M., Becht, P., and Tenhaken, R. 2006. Isolation of a novel ABC-transporter gene from soybean induced by salicylic acid. J. Exp. Bot. 57:2193-2201.

Felsenstein, J. 1985. Confidence limits on phylogenies: An approach using the bootstrap. Evolution 39:783-791.

Geisler, M., Blakeslee, J. J., Bouchard, R., Lee, O. R., Vincenzetti, V., Bandyopadhyay, A., Titapiwatanakun, B., Peer, W. A., Bailly, A., Richards, E. L., Ejendal, K. F., Smith, A.P., Baroux, C., Grossniklaus, U., Müller, A., Hrycyna, C. A., Dudler, R., Murphy, A. S., and Martinoia, E. 2005. Cellular efflux of auxin catalyzed by the Arabidopsis MDR/PGP transporter AtPGP1. Plant J. 44:179-194.

Goodman, C. D., Casati, P., and Walbot, V. 2004. A multidrug resistanceassociated protein involved in anthocyanin transport in Zea mays. Plant Cell 16:1812-1826.

Ito, H., and Gray, W. M. 2006. A gain-of-function mutation in the Arabidopsis pleiotropic drug resistance transporter PDR9 confers resistance to auxinic herbicides. Plant Physiol. 142:63-74.

Jasinski, M., Stukkens, Y., Degand, H., Purnelle, B., Marchand-Brynaert,
J. and Boutry, M. 2001. A plant plasma membrane ABC-type transporter is involved in antifungal terpenoid excretion. Plant Cell 13:10951107.

Jasinski, M., Ducos, E., Martinoia, E., and Boutry, M. 2003. The ATPbinding cassette transporters: Structure, function, and gene family comparison between rice and Arabidopsis. Plant Physiol. 131:1169-1177.

Journet, E. P., van Tuinen, D., Gouzy, J., Crespeau, H., Carreau, V., Farmer, M. J., Niebel, A., Schiex, T., Jaillon, O., Chatagnier, O., Godiard, L., Micheli, F., Kahn, D., Gianinazzi-Pearson, V., and Gamas, P. 2002. Exploring root symbiotic programs in the model legume Medicago truncatula using EST analysis. Nucleic Acids Res. 30:5579-5592. Erratum in: Nucleic Acids Res. 31:1803.

Kamphuis, L. G., Lichtenzveig, J., Oliver, R. P., and Ellwood, S. R. 2008. Two alternative recessive quantitative trait loci influence resistance to spring black stem and leaf spot in Medicago truncatula. BMC Plant Biol. 26:8-30.

Kim, D. Y., Bovet, L., Maeshima, M., Martinoia, E., and Lee, Y. 2007. The ABC transporter AtPDR8 is a cadmium extrusion pump conferring heavy metal resistance. Plant J. 50:207-218.

Kobae, Y., Sekino, T., Yoshioka, H., Nakagawa, T., Martinoia, E., and Maeshima, M. 2006. Loss of AtPDR8, a plasma membrane ABC transporter of Arabidopsis thaliana, causes hypersensitive cell death upon pathogen infection. Plant Cell Physiol. 47:309-318.

Li, Z. S., Alfenito, M., Rea, P. A., Walbot, V., and Dixon, R. A. 1997. Vacuolar uptake of the phytoalexin medicarpin by the glutathione conjugate pump. Phytochemistry 45:689-693.

Marinova, K., Pourcel, L., Weder, B., Schwarz, M., Barron, D., Routaboul, J. M., Debeaujon, I., and Klein, M. 2007. The Arabidopsis MATE transporter TT12 acts as a vacuolar flavonoid/H+ -antiporter active in proanthocyanidin-accumulating cells of the seed coat. Plant Cell 19:20232038

Meade, H. M., Long, S. R., Ruvkun, G. B., Brown, S. E., and Ausubel, F. M. 1982. Physical and genetic characterization of symbiotic and auxotrophic mutants of Rhizobium meliloti induced by transposon Tn5 mutagenesis. J. Bacteriol. 149:114-122.

Medicago Genome Sequencing Consortium. 2006 Medicago truncatula genome sequence: Release 1.0. Norman, OK, U.S.A.

Morsomme, P., Dambly, S., Maudoux, O., and Boutry, M. 1998. Single point mutations distributed in 10 soluble and membrane regions of the Nicotiana plumbaginifolia plasma membrane PMA2 $\mathrm{H}^{+}$-ATPase activate the enzyme and modify the structure of the $\mathrm{C}$-terminal region. J. Biol. Chem. 273:34837-34842.

Rasmussen, R. 2001. Quantitation on the lightcycler. Pages 21-34 in: Rapid Cycle Real-Time PCR-Methods and Applications. S. Meuer, C. Wittwer, and K.-I. Nakagawara, eds. Springer Press, Heidelberg, Germany.

Rea, P. A. 2007. Plant ATP-binding cassette transporters. Annu. Rev. Plant Biol. 58:347-375.

Sanchez-Fernandez, R., Davies, T. G., Coleman, J. O., and Rea, P. A. 2001. The Arabidopsis thaliana ABC protein superfamily: A complete inventory. J. Biol. Chem. 276:30231-30244.

Santelia, D., Vincenzetti, V., Azzarello, E., Bovet, L., Fukao, Y., Düchtig, P., Mancuso, S., Martinoia, E., and Geisler, M. 2005. MDR-like ABC transporter AtPGP4 is involved in auxin-mediated lateral root and root hair development. FEBS (Fed. Eur. Biochem. Soc.) Lett. 579:53995406.

Sasabe, M., Toyoda, K., Shiraishi, T., Inagaki, Y., and Ichinose, Y. 2002. cDNA cloning and characterization of tobacco ABC transporter: $\mathrm{NtPDR} 1$ is a novel elicitor-responsive gene. FEBS (Fed. Eur. Biochem. Soc.) Lett. 518:164-168.

Shitan, N., Bazin, I., Dan, K., Obata, K., Kigawa, K., Ueda, K., Sato, F., Forestier, C., and Yazaki, K. 2003. Involvement of CjMDR1, a plant multidrug-resistance-type ATP-binding cassette protein, in alkaloid transport in Coptis japonica. Proc. Natl. Acad. Sci. U.S.A. 100(2):751756.

Smart, C. C., and Fleming, A. J. 1996. Hormonal and environmental regulation of a plant PDR5-like ABC transporter. J. Biol. Chem. 271:1935119357.

Stein, M., Dittgen, J., Sánchez-Rodríguez, C., Hou, B. H., Molina, A., Schulze-Lefert, P., Lipka, V., and Somerville, S. 2006. Arabidopsis PEN3/PDR8, an ATP binding cassette transporter, contributes to nonhost resistance to inappropriate pathogens that enter by direct penetration. Plant Cell 18:731-746.

Stukkens, Y., Bultreys, A., Grec, S., Trombik, T., Vanham, D., and Boutry, M. 2005. NpPDR1, a pleiotropic drug resistance-type ATP-binding cassette transporter from Nicotiana plumbaginifolia, plays a major role in plant pathogen defense. Plant Physiol. 139:341-352.

Sugiyama, A., Shitan, N., Sato, S., Nakamura, Y., Tabata, S., and Yazaki, K. 2006. Genome-wide analysis of ATP-binding cassette (ABC) proteins in a model legume plant, Lotus japonicus: Comparison with Arab- 
idopsis ABC protein family. DNA Res. 13:205-228.

Sugiyama, A., Shitan, N., and Yazaki, K. 2007. Involvement of a soybean ATP-binding cassette-type transporter in the secretion of genistein, a signal flavonoid in legume-Rhizobium symbiosis. Plant Physiol. 144:2000-2008.

Terasaka, K., Blakeslee, J. J., Titapiwatanakun, B., Peer, W. A., Bandyopadhyay, A., Makam, S. N., Lee, O. R., Richards, E. L., Murphy, A. S., Sato, F., and Yazaki, K. 2005. PGP4, an ATP binding cassette P-glycoprotein, catalyzes auxin transport in Arabidopsis thaliana roots. Plant Cell 17:2922-2939.

Thompson, J. D., Higgins, D. G., and Gibson, T. J. 1994. CLUSTAL W: Improving the sensitivity of progressive multiple sequence alignment through sequence weighting, position-specific gap penalties and weight matrix choice. Nucleic Acids Res. 22:4673-4680.

Tivoli, B., Baranger, A., Sivasithamparam, K., and Barbetti, M. J. 2006. Annual Medicago: From a model crop challenged by a spectrum of necrotrophic pathogens to a model plant to explore the nature of disease resistance. Ann. Bot. (Lond). 98:1117-1128.

Trombik, T., Jasinski, M., Crouzet, J., and Boutry M. 2008. Identification of a cluster IV pleiotropic drug resistance transporter gene expressed in the style of Nicotiana plumbaginifolia. Plant Mol. Biol. 66:165-175.

van den Brûle, S., and Smart, C.C. 2002. The plant PDR family of ABC transporters. Planta 216:95-106.

Verrier, P. J., Bird, D., Burla, B., Dassa, E., Forestier, C., Geisler, M., Klein, M., Kolukisaoglu, U., Lee, Y., Martinoia, E., Murphy, A., Rea, P. A., Samuels, L., Schulz, B., Spalding, E. J., Yazaki, K., and Theodoulou, F. L. 2008. Plant ABC proteins-A unified nomenclature and updated inventory. Trends Plant Sci. 13:151-159.

Yazaki, K. 2005. Transporters of secondary metabolites. Curr. Opin. Plant Biol. 8:301-307.

Zimmermann, P., Hirsch-Hoffmann, M., Hennig, L., and Gruissem, W. 2004. GENEVESTIGATOR. Arabidopsis microarray database and analysis toolbox. Plant Physiol. 136:2621-2632.

\section{AUTHOR-RECOMMENDED INTERNET RESOURCES}

J. Craig Venter Institute EST database: www.tigr.org

Medicago EST Navigation System website: medicago.toulouse.inra.fr/Mt/EST

The Medicago Genome Sequence Consortium Mt2.0 release: www.medicago.org/genome/downloads/Mt2

The German Research Center for Environmental Health Medicago genome database: mips.gsf.de/proj/plant/jsf/medi/index.jsp 